\title{
AMYLOIDOSIS IN A PATIENT WITH RHEUMATOID ARTHRITIS IN THE ERA OF BIOLOGICAL AND JAK INHIBITORS TREATMENT - WE STILL NEED TO BE AWARE
}

Isabela Ohki Nacaguma1, ${ }^{1, \star}$, Heloisa Rodrigues Silva Catalá1, Isadora Medina', Edgard Torres dos Reis Neto'

1.Universidade Federal de São Paulo, São Paulo (SP), Brazil.

*Corresponding author: isabelaohki@gmail.com

\section{BACKGROUND}

Secondary amyloid A (AA) amyloidosis is a late and serious complication of poorly controlled, chronic inflammatory diseases. Rheumatoid arthritis (RA) patients with inadequate response to treatment and longstanding disease with extra-articular manifestations are under risk of developing AA amyloidosis. Although new drugs have proven to be significantly effective in the treatment of RA-related AA amyloidosis, no treatment modality has proven to be ideal. We report a case of a patient refractory to multiple biologics and a JAK inhibitor who evolved with kidney amyloidosis.

\section{CASE REPORT}

A 65-year-old female, with previous diagnosis of RA 10 years ago (symmetric polyarthritis of big and small joints, scleritis, anterior uveitis, bone erosions on X-ray, positive rheumatoid factor and anti-CCP) who was previously treated with conventional disease modifying anti-rheumatic drugs (DMARDs), including methotrexate (suspended because of an allergic reaction), leflunomide (suspended due to increased blood pressure and lack of response). The patient also evolves with primary failure to adalimumab and secondary failure to etanercept. After, tocilizumab and later tofacitinib were initiated, both with secondary treatment failure. During treatment with tofacitinib, the patient presented severe disease activity with polyarthritis (DAS28 5.6) and 24-hour proteinuria of $2.3 \mathrm{~g}$. Renal biopsy demonstrated arteriolar and mesangial glomerular deposits positive in the Congo red test, compatible with secondary amyloid A (AA) amyloidosis (Fig. 1). Due to disease activity and refractoriness to previous described medications, therapy with rituximab was started. Three months after the administration of rituximab, the patient was hospitalized with anterior uveitis and polyarthritis and abatacept was prescribed.

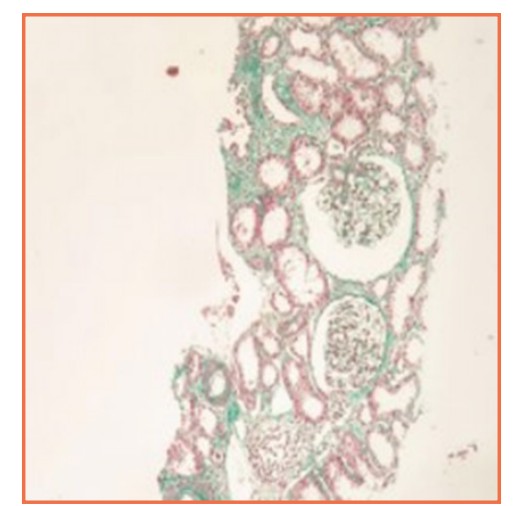

Figure 1. Congo red stain performed on kidney biopsy, positive for arteriolar and mesangial glomerular deposits, compatible with secondary amyloid $A(A A)$ amyloidosis.

\section{CONCLUSION}

The introduction of biological therapies aimed at specific inflammatory mediators has revolutionized the treatment of RA, reducing the incidence of cases with extra-articular manifestations and complications of the disease, including AA amyloidosis. Despite this, in refractory cases, including those with biological therapy, we should be aware of it for the appearance of rare RA complications, such as AA amyloidosis. 